lan C. Bickle, MB, BCh, BAO, FRCR

\section{Department of Radiology} RIPAS Hospital,

Bandar Seri Begawan

Brunei Darussalam
Correspondence: Dr. lan C. Bickle

Consultant Radiologist

Department of Radiology

RIPAS Hospital

Bandar Seri Begawan BA1710

Brunei Darussalam

Phone: + 006738612182

Fax: + 006732242690

Email: firbeckkona@gmail.com

Reprints will not be available from the author.

The author declared that this represents original material that is not being considered for publication or has not been published or accepted for publication elsewhere, in full or in part, in print or electronic media; that the manuscript has been read and approved by the author, that the requirements for authorship have been met by the author, and that the author believes that the manuscript represents honest work.

Disclosures: The author signed disclosures that there are no financial or other (including personal) relationships, intellectual passion, political or religious beliefs, and institutional affiliations that might lead to a conflict of interest.

\section{Sensorineural Hearing Loss: What Lies Beneath? Neurovascular Conflict Secondary to a Dural Arteriovenous Malformation}

This middle-aged gentleman with no previous medical history presented to the local ENT outpatient clinic complaining of right-sided hearing loss. No history of trauma or previous head and neck surgery was elicited.

Following clinical and auditory assessment a right sensorineural hearing loss was confirmed. A right-sided facial palsy was additionally identified on examination.

A MRI of the internal auditory meati was performed (Figure 1A \& 1B). Following radiologist review, MRI and MRA of the brain was undertaken.
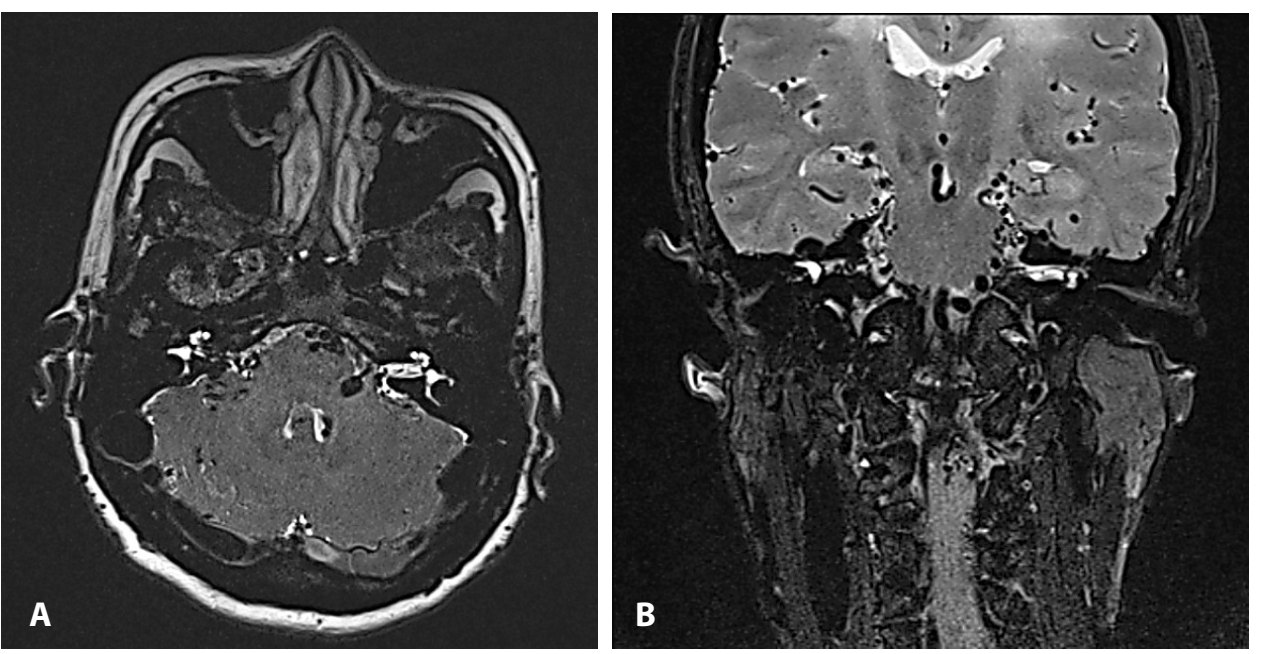

Figures 1A \& B. Axial and Coronal T2 sequences of the IAMS

\section{DISCUSSION}

Auditory impairment is a condition with a legion of potential causes. One of the routine aspects of the assessment process for those with sensorineural hearing loss is MR imaging (MRI) of the internal auditory meati (IAMS).

The vast majority of MRI studies are normal, however one of the more commonly identified pathologies are cerebrovascular abnormalities. The most-well recognised is neurovascular conflict of the vestibulocochlear nerve by a vascular loop at the root entry zone (REZ), however a broader range of potential responsible structural abnormalities 
are known. A wide range of processes for auditory dysfunction have been outlined.' These include: cerebral ischemia events, subarachnoid hemorrhage, cerebrovascular malformations and rarely dural arteriovenous fistulas (dAVFs).

Dural AVF's are abnormal vascular communications between the dural venous sinuses and an arter(ies) - most frequently branches of the external carotid artery. Sensorineural hearing impairment is one of the rarer presenting symptoms. The mechanism for hearing impairment is believed to result from either direct vascular compression on the vestibulocochlear nerve from an enlarged aberrant draining vein or from a vascular steal phenomenon (Figures $2 A \& 2 B$ ). An engorged draining vein from the dAVF causing mechanical compression on the nerve is the most well recognized. ${ }^{2} \mathrm{~A}$ single prior case has been reported of compression from an intraossesous dAVF of the skull base. ${ }^{3}$
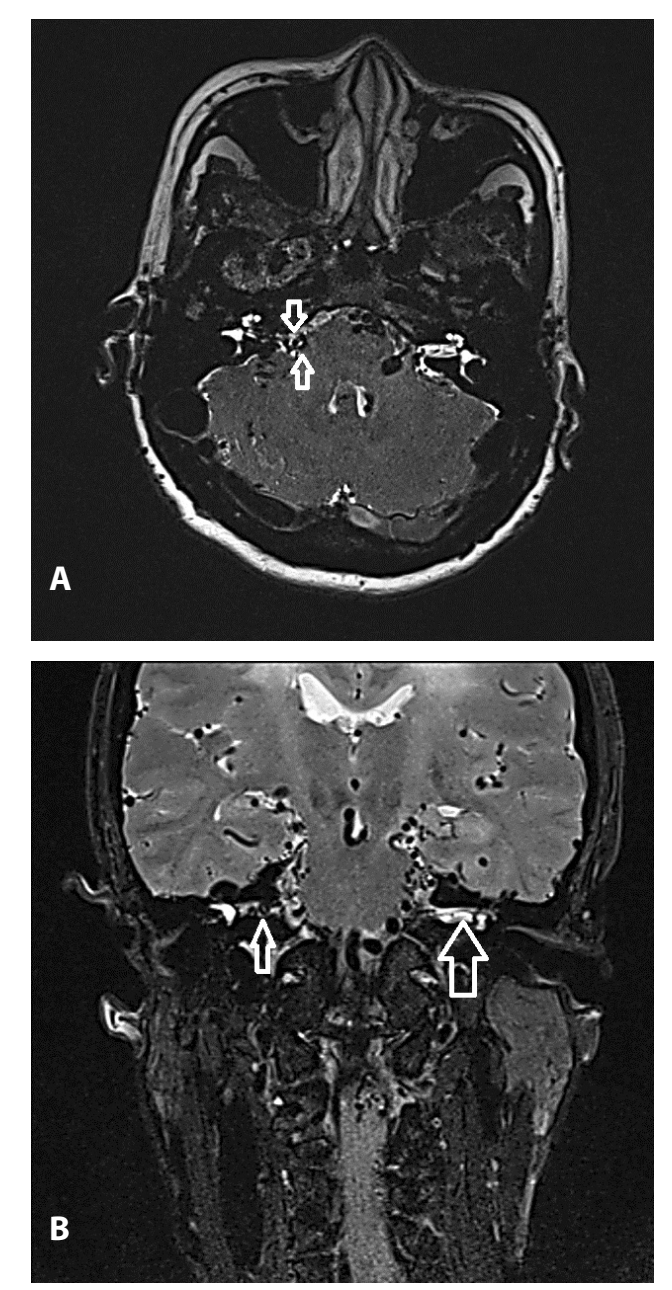

Figures 2A \& B. Axial and Coronal T2 sequences of the IAMS. A: engorged aberrant veins crowding the right IAM (white arrows). B: Engorged veins compressing the intracanalicular right vestibulocochlear nerve (thin white arrow). Normal left side for comparison (thick white arrow)
The arteriovenous fistula may be directly identified (Figure 3) along with the associated signs of enlarged cerebral cortical veins and white matter change of venous hypertension (Figure 4).

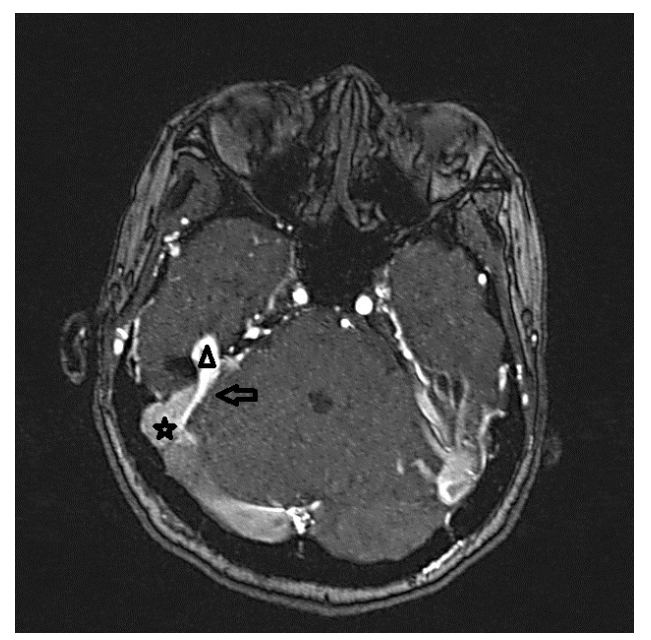

Figure 3. Axial MRA Brain raw data set: A direct communication (arrow) between a distal external carotid artery branch (triangle) and the sigmoid-tranverse sinus junction (star) in keeping with a dural AV fistula

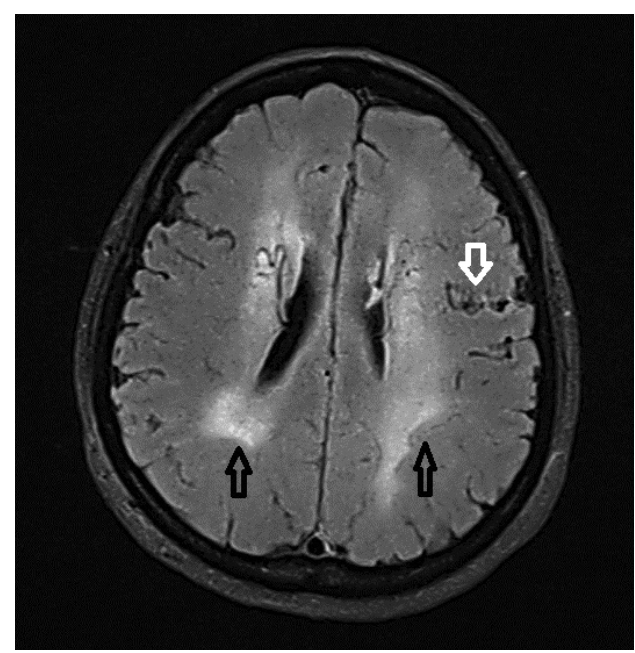

Figure 4. Axial MRI FLAIR Brain: Extensive periventricular high signal (black arrows) due to cerebral venous hypertension. Distended cerebral cortical veins are also present (white arrow) 